\title{
Shock-triggered formation of magnetically-dominated clouds
}

\author{
S. Van Loo ${ }^{1}$, S. A. E. G. Falle ${ }^{2}$, T. W. Hartquist ${ }^{1}$, and T. J. T. Moore ${ }^{3}$ \\ 1 School of Physics and Astronomy, University of Leeds, Leeds LS2 9JT, UK \\ e-mail: svenvl@ast.leeds.ac.uk \\ 2 School of Mathematics, University of Leeds, Leeds LS2 9JT, UK \\ 3 Astrophysics Research Institute, Liverpool John Moores University, Birkenhead, CH41 1LD, UK
}

Received 7 March 2007 / Accepted 4 June 2007

\begin{abstract}
Aims. Our aim is to understand the formation of a magnetically dominated molecular cloud out of an atomic cloud. Methods. A thermally stable warm atomic cloud is initially in static equilibrium with the surrounding hot ionised gas. A shock propagating through the hot medium interacts with the cloud. We follow the dynamical evolution of the cloud with a time-dependent axisymmetric magnetohydrodynamic code.

Results. As a fast-mode shock propagates through the cloud, the gas behind it becomes thermally unstable. The $\beta$ value of the gas also becomes much smaller than the initial value of order unity. These conditions are ideal for magnetohydrodynamic waves to produce high-density clumps embedded in a rarefied warm medium. A slow-mode shock follows the fast-mode shock. Behind this shock a dense shell forms, which subsequently fragments. This is a primary region for the formation of massive stars. Our simulations show that only weak and moderate-strength shocks can form cold clouds which have properties typical of giant molecular clouds.
\end{abstract}

Key words. magnetohydrodynamics (MHD) - shock waves - ISM: clouds - stars: formation

\section{Introduction}

Observations of giant molecular clouds (GMCs) show that they are magnetically dominated (e.g. Crutcher 1999) with values of the ratio $\beta$ of thermal gas pressure to magnetic pressure of roughly 0.04 . Furthermore molecular emission lines show a non-thermal broadening component comparable to the Alfvén speed (Arons \& Max 1975). However, on scales larger than the distances between molecular clouds, the thermal and magnetic pressure are about equal. This means that the formation mechanism of the GMCs must reduce the value of $\beta$ considerably.

While older stellar associations are devoid of molecular gas, molecular clouds in the Solar neighbourhood that do not harbour any young stars, are rare. This suggests that the time lag between the formation of the clouds and the stars must be short. GMCs are thus likely to be transient and dynamically evolving structures (e.g. Hartmann et al. 2001). They can be formed by compressive motions of gravitational and/or turbulent origin (Ballesteros-Paredes et al. 2007). It is thought that the formation of GMCs on scales of many tens to hundreds of parsecs is regulated by the ram pressure from supersonic flows such as supernova remnants, superbubbles and winds of massive stars.

Several groups (e.g. Klein et al. 1994; Mac Low et al. 1994; Fragile et al. 2005) studied the interaction of a strong shock with a cloud. However, these studies do not concentrate on the formation of molecular clouds. Instead, they follow the destruction of the cloud and its effect on the properties of the interstellar medium (ISM). Lim et al. (2005; hereafter LFH05) examined the formation of molecular clouds by the response of a warm atomic cloud to an increase in the pressure of the surrounding medium. They implicitly assumed that this pressure increase is caused by either flow convergences or weak shocks. Their results show that a process relying on pressure-driven compression and radiative cooling leads to the formation of highly magnetically dominated regions within a molecular cloud consistent with observations of GMCs (Audit \& Hennebelle 2005).

In this paper we study the transition of a cloud, triggered by a weak shock, from the warm atomic phase to the cold molecular one. In Sect. 2 we specify the initial conditions of our model and summarise the computational details. Then, we describe the dynamical evolution of the cloud (Sect. 3) and present our results (Sects. 4 and 5). Finally, we discuss these results and give our conclusions in Sect. 6.

\section{The model}

\subsection{Initial conditions}

Like LFH05, we assume that a cloud is initially in the thermally stable warm phase and in pressure equilibrium with the surrounding hot ionised gas. The hot gas itself is not in thermal equilibrium as it is continuously reheated by e.g. supernova explosions and superbubbles. Therefore, the net cooling time is long. For simplicity, we assume that the gas behaves adiabatically. Within the cloud, however, heating and radiative cooling are important.

To describe the thermal behaviour of the gas in the cloud, we adopt a simplified piece-wise power-law cooling function and a constant background heating rate consistent with the standard equilibrium phase diagram (i.e. thermal pressure versus density) of Wolfire et al. (1995). The net heating rate per unit volume is given by

$H=\rho[0.015-\rho \Lambda(T)] \operatorname{erg~cm}{ }^{-3} \mathrm{~s}^{-1}$, 
Table 1. Shock mach number for the numerical models.

\begin{tabular}{cc}
\hline \hline Model & Shock mach number \\
\hline $\mathrm{A}$ & 2.5 \\
$\mathrm{~B}$ & 1.5 \\
$\mathrm{C}$ & 5.0 \\
$\mathrm{D}^{a}$ & 2.5 \\
$\mathrm{E}^{a}$ & 2.5
\end{tabular}

${ }^{a}$ Medium and large cloud models. More details are given in Sect. 5.

where

$$
\Lambda(T)=\left\{\begin{array}{lr}
3.564 \times 10^{16} T^{2.12} & 0 \mathrm{~K} \leq T<141 \mathrm{~K} \\
9.1 \times 10^{18} T & 141 \mathrm{~K} \leq T<313 \mathrm{~K} \\
1.14 \times 10^{20} T^{0.56} & 313 \mathrm{~K} \leq T<6102 \mathrm{~K} \\
1.924 \times 10^{8} T^{3.67} & 6102 \mathrm{~K} \leq T<10^{5} \mathrm{~K} \\
1.362 \times 10^{29} T^{-0.5} & T \geq 10^{5} \mathrm{~K}
\end{array}\right.
$$

(Sánchez-Salcedo et al. 2002; LFH05). For this thermal model, the warm phase does not exist for a number density and thermal pressure higher than $0.5 \mathrm{~cm}^{-3}$ and $3051 k$, respectively. Here, $k$ is the Boltzmann constant.

In our calculations, a cloud with initial radius $R_{\mathrm{cl}}=200 \mathrm{pc}$ has a number density of $0.45 \mathrm{~cm}^{-3}$. As the cloud is in the warm phase, its pressure and temperature, respectively, correspond to $2825 k$ and $6277 \mathrm{~K}$. The surrounding hot gas has a number density of $0.01 \mathrm{~cm}^{-3}$. (Note that these values are the same as in LFH05.) To ensure pressure equilibrium between the cloud and its surroundings, the temperature of the external medium is set to $282500 \mathrm{~K}$. The initial magnetic field is uniform and in the axial direction. It has a value such that the ratio of thermal pressure to magnetic pressure is unity inside the cloud.

While LFH05 examined a cloud subjected to a sudden increase in the external pressure, we consider a steady, planar shock hitting the quiescent cloud. This is known as the smallcloud approximation (see Klein et al. 1994). This intercloud shock propagates along the magnetic field lines through the hot ionised gas at a speed $v_{\text {ext }}$ (quantified by its Mach number $M \equiv v_{\text {ext }} / c_{\mathrm{s}}$, where $c_{\mathrm{s}}$ is the nonmagnetic adiabatic sound speed). Table 1 lists the shock Mach number for each model. We include models $\mathrm{D}$ and $\mathrm{E}$ in order to examine how deviations from the small-cloud approximation affect the results.

\subsection{Computational details}

All of our calculations were done with an adaptive mesh refinement code solving the ideal magnetohydrodynamic (MHD) equations for an axisymmetric geometry (Falle \& Giddings 1993). The code uses an hierarchy of $n$ levels such that the mesh spacing is $\Delta x / 2^{n}$, where $\Delta x$ is the spacing of the coarsest grid. The code refines the grid only where higher resolution is necessary. The basic algorithm is a second-order Godunov scheme (Falle 1991) with a linear Riemann solver and a divergence cleaning algorithm (Dedner et al. 2002).

The computational domain is $0 \leq r / R_{\mathrm{cl}} \leq 2$ and $-2 \leq$ $z / R_{\mathrm{cl}} \leq 3$. The shock propagates in the negative $z$-direction and we impose fixed boundary conditions, i.e. the shock's downstream values, on the $z / R_{\mathrm{cl}}=3$ plane. The other boundary conditions are symmetry on the axis and free flow everywhere else. There are six levels of grids with the finest being $1280 \times 3200$ which gives a mesh spacing of $0.31 \mathrm{pc}$ for our models. Although this resolution is high enough to show the onset of the formation of dense clumps in the interstellar cloud, it is insufficient to follow the complete evolution of the cloud.

\section{Dynamical evolution}

Although the dynamical evolution of the cloud depends on the initial shock Mach number, it is similar for all our models. The results differ only in detail. Therefore, it is useful to describe the evolution before presenting the numerical results in Sects. 4 and 5 .

\subsection{The flow outside the cloud}

When the intercloud shock first hits the cloud, it transmits a shock into the cloud and reflects a shock in the previously shocked intercloud medium. The reflected shock eventually forms a bow shock (or bow wave) in front of the cloud, reducing the flow speed of the incoming gas. After a shock-crossing time $t_{\mathrm{sc}}=2 R_{\mathrm{cl}} / v_{\mathrm{ext}}$, the intercloud shock has swept around the cloud and converged on the axis behind it. Since the curved, converging part of the intercloud shock is moving at a lower speed than the straight part, swept-up material is raised to a lower pressure. The transmitted shock is, therefore, weaker at the sides of the cloud than on the front and back. As a consequence, the shock mainly reduces the cloud size in the direction parallel to the distant upstream flow velocity making it oblate. Eventually, the cloud ends up as a thin disk. The ram pressure of the external flow at the front of the cloud even accelerates this process.

The transmitted shock has a characteristic speed $v_{\text {int }}=$ $v_{\text {ext }} / \chi^{1 / 2}$ where $\chi$ is the density ratio of cloud/intercloud gas. This is considerably lower than the propagation speed of the intercloud shock. Hence, a velocity shear layer forms at the edge of the cloud. This slip surface rolls up to produce a vortex ring sweeping material away from the cloud and is also subject to Kelvin-Helmholtz instability (Klein et al. 1994). However, linear instability analysis (e.g. Chandrasekhar 1961) predicts that a magnetic field strongly suppresses the onset of Kelvin-Helmholtz instability. This is also the case for RayleighTaylor instability that is excited at the cloud surface due to the acceleration of the cloud by the rarefied surrounding gas. Although the inhibition of these instabilities limits the disintegration of the cloud (as seen in hydrodynamic simulations), some fragments still get torn off which survive as small-scale coherent structures (MacLow et al. 1994).

Because the external flow is diverted around the cloud, the magnetic field structure changes significantly. When the shock engulfs the cloud, the intercloud gas near the most upstream point of contact with the cloud is swept around the cloud and carries the magnetic field with it. Since the magnetic field lines are anchored in the cloud, they are stretched into a self-reversed layer (Mac Low et al. 1994). Eventually, these lines double over and form a region of strong reversed field. This region may be subject to magnetic reconnection.

\subsection{The flow inside the cloud}

A fast-mode shock and a trailing slow-mode shock are driven into the cloud. The fast-mode shock compresses the gas so that it is in the thermally unstable temperature range. As the time scale for the radiative cooling is much shorter than the time for the shock to be reflected at the axis, given by the cloud-crushing time $t_{\mathrm{cc}}=R_{\mathrm{cl}} / v_{\text {int }}$, the gas loses a significant fraction of its internal energy during the compression. This initiates the formation of cold dense regions embedded in a diffuse warm medium. These clumps continue to cool and accumulate material until the cooling balances the heating. The clumps end up in approximate pressure equilibrium with their surroundings. The number 

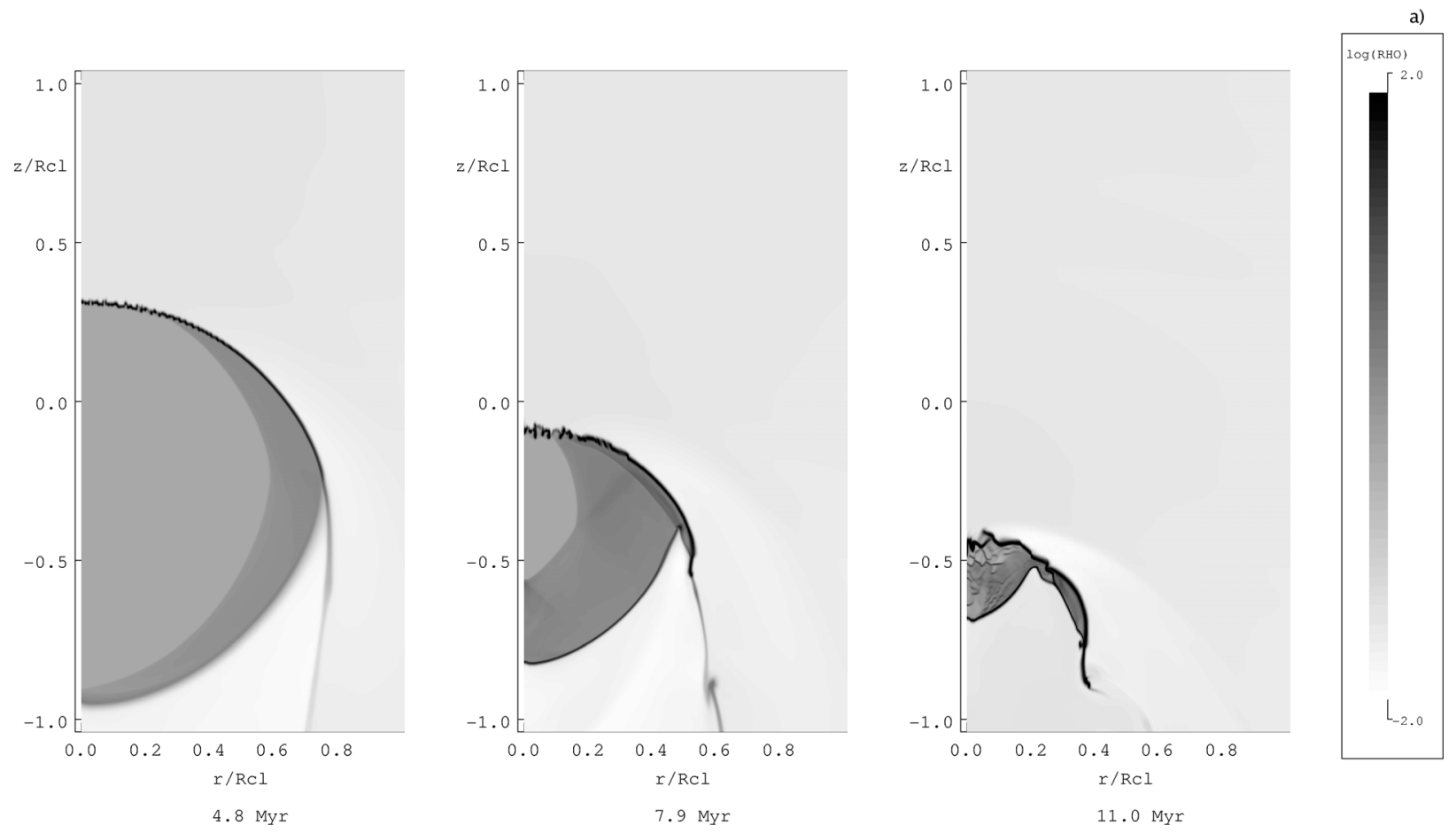

Fig. 1. Grey-scale plots of the number density (logarithmic, a)) and $\beta$ (linear, b)) at the times shown for model A. For the number density the range is $-2-2$ and for $\beta$ it is $0-1$. The size of the computational box shown is $0 \leq r / R_{\mathrm{cl}} \leq 1$ and $-1 \leq z / R_{\mathrm{cl}} \leq 1$. The post-shock flow is in the negative $z$-direction.

density of a clump is then in the range $500-1000 \mathrm{~cm}^{-3}$ which is typical for translucent clumps in GMCs (e.g. Williams et al. 1995).

Furthermore, as the magnetic pressure increases across the fast-mode shock, the value of $\beta$ falls below unity. This provides the ideal conditions for the generation, by MHD waves, of dense cores within these clumps (e.g. Falle \& Hartquist 2002; Van Loo et al. 2006), especially as thermal instability produces a supersonic velocity dispersion within the cloud (Koyama \& Inutsuka 2002). However, we do not have sufficient spatial resolution to follow the complete clump and dense core formation process. Moreover, self-gravity, which is not included in our models, becomes dynamically important once some dense cores are compressed to their measured number densities.

The slow-mode shock moves much more slowly than the fast-mode shock. The magnetic pressure decreases behind this shock, and consequently there is nothing to stop the gas from compressing while it cools. Hence, a shell of cold, dense gas forms close to the edge of the cloud. The dense shell first develops on the upstream parts of the cloud, even before the shock has interacted with the rest of the cloud. Furthermore, the velocity shear at the surface of the cloud leads to a broader dense shell near the stagnation point (see e.g. Fig. 1) as the gas compressed by the slow-mode shock is swept around the cloud. The thickness of this shell thus depends on the level of shear at the edge, i.e. the shock Mach number.

The interaction of the shock with the cloud produces dense regions in the cloud, i.e. a shell at the edge of the cloud and several individual clumps within the cloud. The shell contains a large fraction of the cloud's total mass. It is also unstable due to thermal instability and dynamical instabilities (such as Kelvin-Helmholtz and Rayleigh-Taylor), so that it fragments into cold, massive filaments. Our simulations show this fragmentation at later times. This means that the shell may well be the precursor of massive star formation. The clumps within the cloud, may form only low-mass stars as they are magnetically dominated.

\section{Numerical results}

Figure 1 shows grey-scale plots of the number density and $\beta$ for model A. We can clearly see that, as the fast-mode shock moves through the cloud, the swept-up gas becomes magnetically dominated, i.e. $\beta$ drops below unity. This is because the thermal gas pressure decreases due to the transition to the thermally unstable phase, while the magnetic pressure increases behind the fastmode shock. It can then be expected that, by the time the shock reflects at the symmetry axis, a significant fraction of the volume of the cloud has a low- $\beta$ value. Figure 2 shows the fraction of cloud volume with $\beta<0.1$ as a function of time for each of three models. At around $t_{\mathrm{cc}}$, the volume fraction of the model A cloud for which $\beta<0.1$ is about $\sim 0.9$. Actually, most of the cloud is in a low- $\beta$ regime for a significant period of time ( $\sim 3 \mathrm{Myr}$ ). Within this time range, the cloud has a characteristic mean number density and radius of $\approx 20 \mathrm{~cm}^{-3}$ and $\approx 50 \mathrm{pc}$. Furthermore, we find that the velocity dispersion of the cloud is highly supersonic. These are all typical properties of a GMC.

Although a small fraction of the volume of cloud is not magnetically dominated, its corresponding mass fraction is considerable (see Fig. 3). About half of the total mass is in the boundary layer of the cloud. Here, the gas is in the cold phase at a high thermal pressure so that $\beta \approx 1$. While the conditions in the interior of the cloud are ideal for the generation of dense clumps and embedded cores by MHD waves, this is not true for the dense shell. It is, however, expected that this shell breaks 

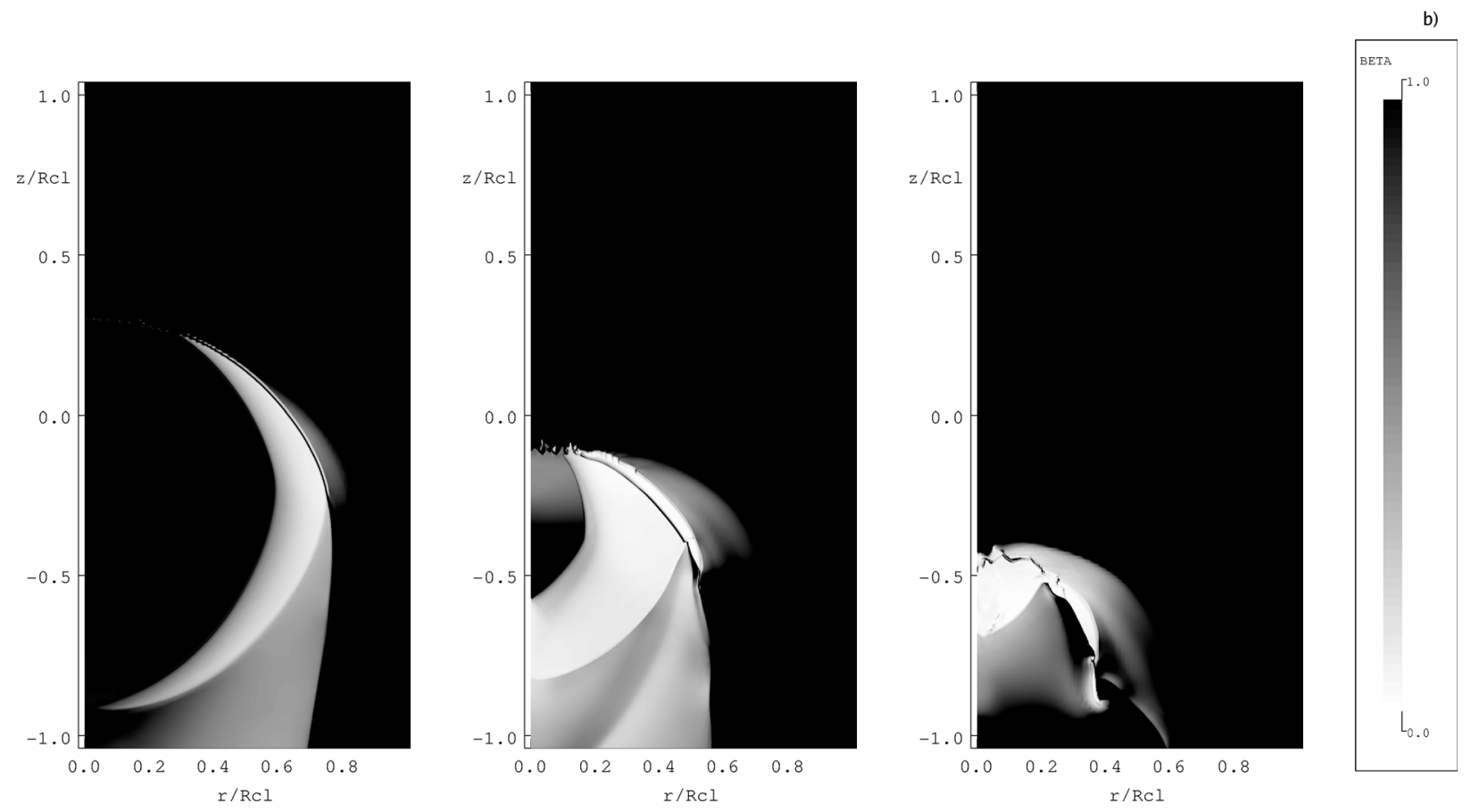

Fig. 1. continued.

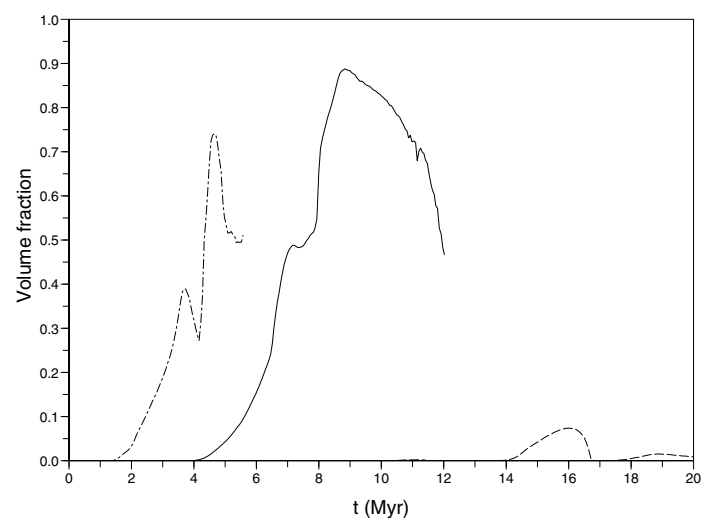

Fig. 2. The fraction of the cloud volume with $\beta<0.1$ as a function of time (in Myr) for Model A (solid), Model B (dashed) and Model C (dashed-dotted).

up into dense fragments (Koyama \& Inutsuka 2000). This fragmentation is observed in our simulations. The densest fragments have number densities of $\sim 10^{3} \mathrm{~cm}^{-3}$ and sizes of $\sim 1 \mathrm{pc}$. With a temperature of about $30 \mathrm{~K}$ for these fragments, we derive a Jeans mass of $\sim 350 M_{\odot}$ which is similar to the inferred mass. (We have assumed a near spherical shape for the dense fragments.) Therefore, the boundary layer may be a site of massive star formation.

For the other models, we find similar results, except that stronger shocks (Model C) lead to a faster evolution (see Fig. 2). This is because the propagation speed, $v_{\text {int }}$, of the shock increases with Mach number, reducing the dynamical time-scale $t_{\mathrm{cc}}$. (The cloud crushing time is $t_{\mathrm{cc}} \approx 4 \mathrm{Myr}$ for Model C, while $t_{\mathrm{cc}} \approx 8.5 \mathrm{Myr}$ for Model A.) Magnetically-dominated regions thus develop on shorter time-scales. However, the compression of the cloud into a disk also proceeds faster due to a higher ram pressure exerted by the external flow (i.e. the ram pressure is

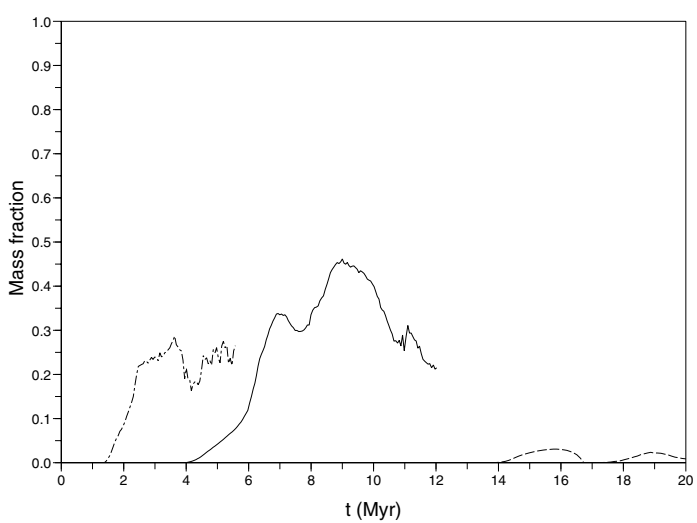

Fig. 3. The fraction of the cloud mass with $\beta<0.1$ as a function of time (in Myr) for Model A (solid), Model B (dashed) and Model C (dasheddotted).

proportional to $M^{2}$ ). Our simulations show that, for Model C, the time-scale for flattening is $\sim 1 t_{\mathrm{cc}}$, while it increases to $\sim 1.5-$ $2 t_{\mathrm{cc}}$ for weaker shocks (Model A and B). Consequently, the lifetime of a molecular cloud produced by a strong shock has an upper limit of a few Myr before it is flattened. The formation of $\mathrm{H}_{2}$ and $\mathrm{CO}$ from atomic gas requires a similar timescale (Bergin et al. 2004; Glover \& Mac Low 2007). Thus, as the dynamical evolution is too rapid for hydrogen to be completely converted to $\mathrm{H}_{2}$, strong shocks do not produce giant molecular clouds. However, molecules can still form in the flattened cloud. As this thin disk is being shredded by instabilities (MacLow et al. 1994), this results in the formation of small molecular clouds with sizes of a few pc.

The time-scale for molecular cloud formation by a weak shock (Model B) is compatible with the expected time-scales for the formation of molecules (the cloud flattens on a timescale of $25 \mathrm{Myr}$ ). However, in this case the fast-mode shock 


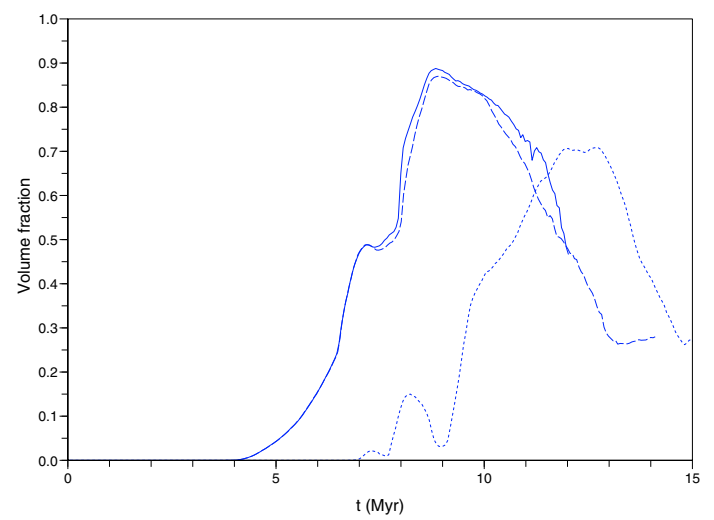

Fig. 4. Similar to Fig. 2, but now for small (solid), medium (dashed) and large (dotted) cloud approximation.

propagating through the cloud does not compress the gas sufficiently to induce fast radiative cooling. As the cooling time of the gas is longer, it is difficult to produce magnetically-dominated regions within the cloud. Figure 2 shows that less than $10 \%$ of the cloud has $\beta<0.1$ during its entire evolution. Note, however, that we have not included any feedback effect of star formation. It is believed to take place in the dense boundary layer of the cloud. We can then expect proto-stellar outflows to reduce the value of $\beta$ locally, thus, forming magnetically dominated regions.

\section{Medium and large clouds}

In the previous section we showed the results for a shock, being driven by a constant distant upstream pressure, interacting with a cloud; i.e. the so-called small-cloud approximation. However, the pressure behind a wind-blown bubble or a supernova blast wave can change significantly on a time-scale shorter than $t_{\mathrm{cc}}$, or even $t_{\mathrm{sc}}$. By comparing these time-scales to the time-scale, $t_{\mathrm{p}}$, of pressure variations we can make a further distinction between medium-sized and large clouds (Klein et al. 1994). For medium-sized clouds, the pressure does not vary much as the shock sweeps around the cloud, but it does change significantly before the cloud is crushed, i.e. $t_{\mathrm{cc}} \geq t_{\mathrm{p}} \geq t_{\mathrm{sc}}$. For large clouds, $t_{\mathrm{p}}<t_{\mathrm{sc}}$, and the pressure thus changes while the shock sweeps around the cloud.

To study the effect of pressure variation behind the shock, we assumed that a shock is accelerated exponentially from $M=1.1$ to a higher value of $M$ (given in Table 1 for models D and E) after which it accelerates no more. In our calculations, we assumed that the period of constant shock strength is $\sim 1$ Myr for a large cloud (Model E) and $~ 5 \mathrm{Myr}$ for a medium-sized cloud (Model D). Our model is a simplified description of a blast wave. However, we prefer this to the implementation of the SedovTaylor blast-wave solution, as we are only interested in the qualitative differences from the small-cloud approximation.

Figure 4 shows that, for medium-sized clouds, the results do not change significantly from the small-cloud approximation. Large clouds, however, evolve at a lower rate towards a low- $\beta$ regime. This can be readily explained by the fact that the pressure changes as the shock sweeps around the cloud so that the shock strength on the upstream and downstream sides is quite different. The fast-mode shock therefore does not produce the same compression of the gas as in the small-cloud approximation. This results in a longer cooling time and a delay in the
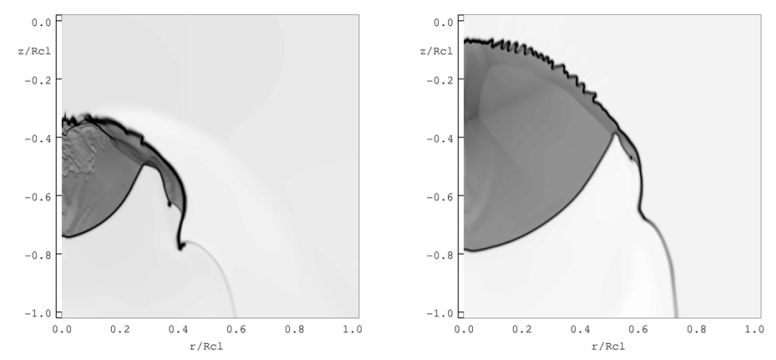

Fig. 5. Logarithmic grey-scale plot of the number density for Model A (left) and Model E (right) at $10 \mathrm{Myr}$. The range for the number density is $-2-2$.

formation of a low- $\beta$ cloud. Yet, most of the cloud in the largecloud approximation still becomes magnetically dominated.

The fact that the pressure behind the shock decreases on time-scales shorter than $t_{\mathrm{sc}}$ has the additional advantage that the cloud is exposed to a lower ram pressure. Consequently, the cloud does not flatten into a disk as fast as in the small-cloud approximation (see Fig. 5). This is relevant as it shows that shocks, stronger than the small-cloud approximation would indicate, can produce clouds resembling GMCs.

\section{Conclusions and discussion}

In the present paper we studied the interaction of a shock with an initially warm, thermally stable cloud of uniform density. As the shock sweeps around the cloud, a transmitted fast-mode shock propagates through the cloud producing magneticallydominated regions behind it. This provides the ideal conditions for MHD waves to produce high-density clumps within the cloud. These clumps may eventually form low-mass stars.

A slow-mode shock follows the fast-mode shock leading to a high-density layer near the boundary of the cloud. As the shock interacts first with the front of the cloud, the dense layer forms there first. The dense layer is subject to instabilities and fragments into massive clumps. Our analysis shows that these clumps are close to gravitationally bound and, thus, are possible precursors of massive stars. This agrees well with the findings that OB stars form almost always near the boundary of molecular clouds and even prefer to form at one particular edge of the cloud (e.g. Blaauw 1962; Elmegreen \& Lada 1977; Israel 1978; Gatley et al. 1979; Fich et al. 1982). Furthermore, the cometary cloud structure seen in our simulations, i.e. a massive head and longspread tail, seems characteristic for clouds harbouring clusterforming cores (Tachihara et al. 2002).

In our simulations we imposed a sharp boundary between the cloud and the surrounding medium. However, real interstellar clouds have an internal density gradient, e.g. the southwest cloud in the Cygnus Loop supernova remnant shows a gradual drop in density near the boundary (Patnaude et al. 2002). Hydrodynamical simulations of the effect of smooth boundaries show that the initial density pattern remains imprinted on the shocked cloud (Nakamura et al. 2006). Preliminary results of MHD simulations show a similar effect, but we still find that a thin dense layer arises near the boundary of the cloud. The effect of a density gradient and the presence of condensations within the cloud will be investigated in detail in a subsequent paper.

A likely example of a shock-cloud interaction is found in the W3 GMC. Figure 6 shows the W3 GMC as traced by ${ }^{12} \mathrm{CO} J=$ $1-0$ emission at 50 arcsec resolution (Bretherton 2003). The cloud is $\sim 60 \mathrm{pc}$ in size with a total gas mass of nearly $4 \times 10^{5} M_{\odot}$, 


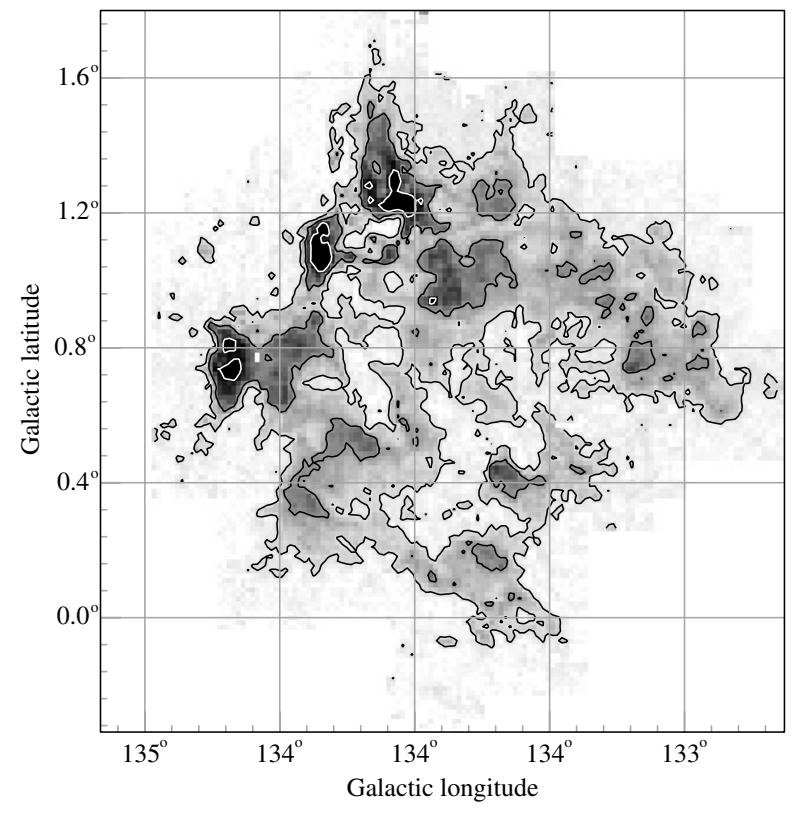

Fig. 6. The $\mathrm{W} 3 \mathrm{GMC}$ in the $J=1-0$ transition of ${ }^{12} \mathrm{CO}$ with a 50 arcsec resolution (Bretherton 2003). High-mass star-forming regions are found in the HDL situated in the North-East of the cloud.

of which $\sim 40 \%$ is in a boundary layer referred to as the highdensity layer (HDL) (Lada et al. 1978). The mean number density in the cloud is around $40 \mathrm{~cm}^{-3}$ implying a very clumpy medium in the cloud. A number of high-mass star-forming regions are found in the HDL which runs parallel to the edge of the W4 H II region and is likely to be formed by the expansion of the $\mathrm{H}$ II region and the stellar winds of the W4 OB association. Recently, Moore et al. (2007) surveyed W3 and found that the fraction of the total cloud mass in dense clumps is as high as 0.26 in the HDL and only 0.05 in the diffuse part of the cloud. These values are consistent with those obtained in our simulations.

Our simulations support the idea that molecular clouds are transient objects. In this view, star formation happens quickly and the parental GMCs are short-lived with observations suggesting lifetimes of the order of 10-20 Myr (see e.g. Blitz et al. 2007). A lower limit of a few Myr is set by the formation of $\mathrm{H}_{2}$ and $\mathrm{CO}$ from the atomic gas (Bergin et al. 2004). Although the lifetimes of the cloud in our simulations can only give a rough guide because the cloud compression into a disk is fastest when the external flow is along the magnetic field lines, they agree well with observed values. For strong shocks $(M \geq 5)$, however, the evolution seems to be too fast to produce giant molecular clouds with morphologies as those observed. Nevertheless, strong shocks can still produce molecular clouds as the formation of molecules continues within the small coherent structures that fragment off the initial cloud.
Our results would also seem to rule out very weak shocks as agents for molecular-cloud formation since they do not produce clumped clouds. A deficiency of our model is that it does not include any feedback from star formation such as proto-stellar winds and jets, and expanding $\mathrm{H}$ II regions. However, following the sequential star formation picture of Elmegreen \& Lada (1977), we argue that these processes occur in the boundary layer of the cloud and inject sufficient energy into the cloud to produce a clumped medium. We, therefore, conclude that only weak to moderate-strength shocks are the likely triggers for the formation of GMCs in the ISM.

Acknowledgements. We thank the anonymous referee for his/her useful comments that improved the original manuscript and J. Urquhart for useful discussions. S.V.L. thanks PPARC for the financial support.

\section{References}

Arons, J., \& Max, C. E. 1975, ApJ, 196, L77

Audit, E., \& Hennebelle, P. 2005, A\&A, 433, 1

Ballesteros-Paredes, J., Klessen, R. S., Mac Low, M.-M., et al. 2007, in Protostars and Planets V, ed. B. Reipurth, D. Wit, K. Keil (Tucson: University of Arizona Press), 63

Bergin, E. A., Hartmann, L. W., Raymond, J. C., \& Ballesteros-Paredes, J. 2004, ApJ, 612, 921

Blaauw, A. 1962, in Interstellar Matter in Galaxies, ed. L. Woltjer (New York: Benjamin), 32

Blitz, L., Fukui, Y., Kawamura, A., et al. 2007, in Protostars and Planets V, ed. B. Reipurth, D. Jewitt, K. Keil (Tucson: University of Arizona Press), 81 Bretherton, D. E. 2003, Ph. D. Thesis, Liverpool John Moores University Chandrasekhar, S. 1961, Hydrodynamic and hydromagnetic stability (London: Oxford University Press)

Crutcher, R. M. 1999, ApJ, 520, 706

Dedner, A., Kemm, F., Kroner, D., et al. 2002, J. Comput. Phys., 175, 645

Elemegreen, B. G.,\& Lada, C. J. 1977, ApJ, 214, 725

Falle, S. A. E. G. 1991, MNRAS, 250, 851

Falle, S. A. E. G., \& Giddings, J. R. 1993, in Numerical Methods for Fluid Dynamics 4, ed. K. W. Morton \& M. J. Baines (Oxford: Clarendon), 335

Falle, S. A. E. G., \& Hartquist, T. W. 2002, MNRAS, 329, 195

Fich, M., Treffers, R. R., \& Blitz, L. 1982, in Regions of Recent Star Formation, ed. R. S. Roger \& P. E. Dewdney (Dordrecht: Reidel), 201

Fragile, P. C., Anninos, P., Gustafson, K., \& Murray, S. D. 2005, ApJ, 619, 327

Gatley, I., Becklin, E. E., Sellgren, K., \& Werner, M. W. 1979, ApJ, 233, 575

Glover, S. C. O., \& Mac Low, M.-M. 2007, ApJS, 169, 239

Israel, F. P. 1978, A\&A 70, 769

Hartmann, L., Ballesteros-Paredes, J., \& Bergin, E. 2001, ApJ, 562, 852

Klein, R. I., McKee, C. F., \& Colella, P. 1994, ApJ, 420, 213

Koyama, H., \& Inutsuka, S.-I. 2000, ApJ, 532, 980

Koyama, H., \& Inutsuka, S.-I. 2002, ApJ, 564, L97

Lada, C. J., Elmegreen, B. G., Cong, H.-I., \& Thaddeus, P. 1978, ApJ, 226, 39

Lim, A. J., Falle, S. A. E. G., \& Hartquist, T. W. 2005, ApJ, 632, L91 (LFH05)

Mac Low, M.-M., McKee, C. F., \& Klein, R. I. 1994, ApJ, 433, 757

Moore, T. J. T., Bretherton, D. E., Fujiyoshi, T., et al. 2007, MNRAS, in press

Nakamura, F., McKee, C. K., Klein, R. I., et al. 2006, ApJS, 164, 477

Patnaude, D. J., Fesen, R. A., Raymond, J. C., et al. 2002, AJ, 124, 2118

Sanchéz-Salcedo, F. J., Vázquez-Semadeni, E., \& Gazol, A. 2002, ApJ, 577, 768

Tachihara, K., Onishi, T., Mizuno, A., \& Fukui, Y. 2002, A\&A, 385, 909

Van Loo, S., Falle, S. A. E. G., \& Hartquist, T. W. 2006, MNRAS, 370, 975

Wolfire, M. G., Hollenbach, D., McKee, C. F., et al. 1995, ApJ, 443, 152

Williams, J. P., Blitz, L. \& Stark, A. A. 1995, ApJ, 451, 252 